\title{
Optical manipulation of airborne particles: techniques and applications $\dagger$
}

\author{
David McGloin, ${ }^{* a b}$ Daniel R. Burnham, ${ }^{a b}$ Michael D. Summers, ${ }^{a}$ \\ Daniel Rudd, ${ }^{a b}$ Neil Dewar ${ }^{a}$ and Suman Anand ${ }^{c}$
}

\author{
Received 12th February 2007, Accepted 17th April 2007 \\ First published as an Advance Article on the web 30th July 2007 \\ DOI: $10.1039 / \mathbf{b 7 0 2 1 5 3 d}$
}

In the following paper, we discuss new methods to trap and manipulate airborne liquid aerosol droplets. We discuss the single gradient force trapping of water aerosols in the 2-14 micron diameter range using both $532 \mathrm{~nm}$ and $1064 \mathrm{~nm}$ light, as well as the holographic optical trapping of arrays of aerosols. Using this holographic technique, we are able to show controlled aerosol coagulation. We also discuss two techniques based on the radiation pressure trapping of aerosols, namely the dual beam fibre trap and the controlled guiding of aerosols using Bessel beams. We conclude with a discussion of new topics for study based upon these techniques and some possible applications.

\section{Introduction}

The ability of light to exert forces on microscopic bodies is encapsulated in the technology known as optical tweezers. ${ }^{1}$ Such devices make use of a tightly focussed laser beam to trap and manipulate particles and have, over the last two decades, enabled a wide range of studies in the physical, chemical and biological sciences to be carried out. ${ }^{2-5}$ Of particular note is that optical tweezers offer a noncontact method to manipulate particles in the size range of a few microns, which typically includes particles such as industrially-relevant colloids, biological cells and, as will be discussed in this paper, aerosols of particular interest to atmospheric scientists.

The beauty of the optical tweezers technique is its relative simplicity, with little more than a laser and a simple microscope system required for basic studies. This has led to a large number of labs throughout the world making use of the unique opportunities to probe particle behaviour, dynamics and composition within the trap. It is fair to say, however, that the vast majority of the experiments carried out to date involve particles immersed in a liquid. Little work has been carried out on airborne particles, primarily due to the relative difficulties associated with airborne trapping, and perhaps the field not being fully appreciated by those working on the dynamics and characterisation of airborne particles.

\footnotetext{
${ }^{a}$ SUPA, School of Physics and Astronomy, University of St. Andrews, St. Andrews, Fife KY16 9SS

${ }^{b}$ Electronic Engineering and Physics Division, University of Dundee, Dundee DD1 4 HN

${ }^{c}$ Polymeric \& Soft Material Section, National Physical Laboratory, Dr. K. S. Krishanan Road, New Delhi, India
}

$\dagger$ The HTML version of this article has been enhanced with colour images. 
In recent years, however, a small number of pilot studies have been carried out looking at how airborne particles, in particular liquid aerosols, interact with optical fields and the type of processes that can be studied using such trapped particles. The first study of using optical tweezers for airborne particles was carried out by Omori et al., ${ }^{6}$ which looked at trapping solid particles in air (and would appear to be the only such study to date) using a non-inverted trapping geometry. All subsequent aerosol studies have used an inverted geometry that uses gravity to aid the trapping process. Six years later, Magome et al. ${ }^{7}$ demonstrated the first use of optical tweezers to trap an aerosol particle (a water droplet produced from a supersaturated vapour of water using a $\mathrm{NH}_{4} \mathrm{Cl}$ microparticle as a nucleation centre). In 2004, two further papers appeared, both carried out in collaboration with Andy Ward's group at the Rutherford Appleton Laboratory (RAL). Hopkins et al. ${ }^{8}$ demonstrated the controlled coagulation of optically trapped aerosol droplets using a dual beam tweezers system and measured the droplet volumes very accurately using Cavity Enhanced Raman Spectroscopy (CERS), while King et al. ${ }^{9}$ examined how seawater and oleic acid droplets reacted with ozone. Jonathan Reid's group have followed up their initial paper with further studies looking at the CERS signals from growing and evaporating droplets, ${ }^{10}$ methods for examining how immiscible aerosols mix when coagulated, ${ }^{11}$ the characterisation of such coagulation using optical tweezers ${ }^{12}$ and a method to compare the growth of more than one aerosol droplet in parallel. Furthermore, our group has carried out studies on making use of holographic optical tweezers to trap many particles simultaneously and coagulate them, ${ }^{13}$ and using radiation pressure to guide droplets over large distances. ${ }^{14}$ Thus, while there is a rather more extended literature dealing with optical levitation, the experimental work carried out on airborne particles using optical tweezers is very limited, and as such should prove to be an area ripe for study.

This paper is intended to highlight some of the technologies that are perhaps well established within the optical tweezers community that can be utilised to trap aerosol particles. We will also discuss some of the practical aspects associated with such techniques and discuss ways in which the technologies could develop, as well as outlining some of the interesting effects we have noticed in carrying out these studies, which run, perhaps, against the grain of conventional thinking with regard to optical tweezers. We will discuss four main experimental ideas: (1) a study of the trapping efficiency of various aerosol particles at both infrared $(1064 \mathrm{~nm})$ and visible wavelengths, (2) the use of holographic optical tweezers techniques to trap, manipulate and coagulate aerosol droplets, (3) the use of a dual beam fibre trap to trap aerosols and (4) the use of laser beams to levitate and guide aerosols over large distances. We will begin with a brief introduction to optical forces.

\section{Physics of optical manipulation}

In the early days of optical manipulation, before the development of a single beam optical tweezers, much use was made of radiation pressure, the force exerted by light through the momentum transfer of photons hitting the surface of a body. Using this technique, one can simply trap objects by balancing them against gravity, and in this early work there were a number of studies looking at aerosol particles. ${ }^{15,16}$ This force can be written as:

$$
F_{\text {scatt }}=\frac{n_{\mathrm{m}}}{c} \frac{128 \pi^{5} r^{6}}{3 \lambda^{3}}\left(\frac{m^{2}-1}{m^{2}+2}\right)^{2} I_{0}
$$

Where $n_{\mathrm{m}}$ is the refractive index of the medium, $c$ is the speed of light, $r$ is the particle radius, $\lambda$ is the laser wavelength, $I_{0}$ the laser intensity and $m=n_{\mathrm{p}} / n_{\mathrm{m}}$, with $n_{\mathrm{p}}$ the refractive index of the particle ( $m$ is referred to as the relative refractive index).

In 1986, Ashkin et al. ${ }^{1}$ published a landmark paper demonstrating the use of a single beam to trap particles by making use of the optical gradient force, which is 
proportional to the intensity gradient of the trapping light (typically a Gaussian laser beam). This force can be written as:

$$
F_{\text {grad }}=\frac{2 \pi r^{3}}{c}\left(\frac{m^{2}-1}{m^{2}+2}\right) \nabla I_{0}
$$

The force is strong enough to overcome the radiation pressure force when the light is focussed down to an extremely small spot, which is typically achieved by making use of a high NA oil immersion microscope objective. The gradient force acts to pull the particles towards the focal point of the beam (both laterally and axially) provided the refractive index of the particle is higher than the surrounding medium, i.e., $m>1$. Once trapped, the particle can be considered as residing in a harmonic potential and as such the particle dynamics can be described by the following equation of motion:

$$
\gamma_{0} \dot{x}(t)+k x(t)=\xi(t)
$$

Where $\gamma_{0}$ is the viscous drag coefficient and $k$ is the trap stiffness. $\xi$ is the stochastic force due to thermal fluctuations. Typically, the motion of the particle is heavily overdamped and the inertial forces can be neglected, as in eqn (3). However, this issue is slightly less clear cut when dealing with particles trapped in air, as the damping of the medium is reduced. Although a typical airborne particle is still in the overdamped regime, it can be coerced towards the underdamped regime. ${ }^{17}$ This fact coupled with the slightly higher-than-normal refractive index difference between the particle and the medium (compared to fluid immersed particles) is one of the likely explanations for the unusual behaviour observed when trapping airborne particles, some of which are discussed below.

\section{Optical trapping technique for aerosols}

Optical manipulation of aerosols poses a few challenges when one compares it with trapping in liquid. The primary challenge is that the process becomes passive as opposed to active. In water-based samples, one can move the laser beam around until a particle is found, i.e., we can actively seek a particle out. In air this is not possible due to the motion of the aerosols. We must passively wait until an aerosol falls into our trap. As such the process can be rather laborious.

Another consideration is that we must control the environment to a greater degree than when dealing with fluid, by taking care of the relative humidity and the vapour pressure of the droplet. ${ }^{8}$ Typically, the humidity can be controlled by placing some fluid into the sealed sample chamber and the vapour pressure of water can be controlled by adding sodium chloride. This has the effect of reducing the vapour pressure and enables the droplets to quickly reach an equilibrium size, allowing stable trapping times of many hours.

Our studies are motivated by the emerging need for tools that can hold and manipulate micrometer, and hopefully smaller, diameter aerosols ${ }^{18}$ so as to study the particle composition, particle size, morphology, phase and mixing state, which all contribute to the chemical and physical state of the aerosol. Such properties include light scattering, toxicity, chemical activity and particle diffusion rates. These in turn are important in a wide range of fields such as atmospheric chemistry and physics, combustion science, dusty plasmas and health science. By probing such droplets we can measure properties such as nucleation rates, mass and heat transfer between the droplet and its surrounding medium and coagulation dynamics.

The development of a controllable optical platform provides an excellent basis for such studies and it is the development of such a platform that we are primarily motivated by here. Principally we will make use of established fluid (i.e., water)based techniques and apply them to airborne particles. These techniques are mainly optical gradient force techniques, that is, based on optical tweezers, but we also develop some new techniques based on radiation pressure, which are 
more widely developed, having received some initial attention by Ashkin in the 1970 s. $^{15,16}$

\section{Aerosol trapping using optical tweezers}

The ability of green laser light $(532 \mathrm{~nm})$ to trap and hold indefinitely single aerosol droplets is now well established., ${ }^{9,13}$ Typically, we can measure axial (in the $z$-direction) $Q$ values (which are a measure of the efficiency of the tweezers, with $Q=1$ being the maximum efficiency) of $Q \sim 0.22$, which compares favourably with conventional fluid immersed particles, where $Q$ s are typically $<0.2$, and often are an order of magnitude lower. The low absorption of water at this wavelength indicates that heating should not be a significant problem and that trapping should be robust and long lasting, which indeed we find to be true. ${ }^{13}$

However, the first paper on the optical trapping of aerosols using the gradient force $^{7}$ used infrared (IR) wavelengths and the suggestion was that while aerosols could be trapped, there was associated heating and the trapping was short lived, indeed the experiment was to investigate this heating effect. In ref. 7, the water droplets were not created using a nebuliser as in subsequent work, but by reacting ammonia and hydrochloric acid to create nucleation sites of ammonium chloride, around which droplets form. It has also been suggested to us $^{19}$ that long term trapping of aerosols using IR light was not possible and that this would limit the usefulness of aerosol tweezers. However the absorption spectra suggest that heating should not be an issue at IR wavelengths and we set out to explore optical trapping at this wavelength to try to refute this suggestion. We made use of a standard inverted tweezers setup, Fig. 1. The advantage of carrying out the experiments at IR wavelengths is that the cost per watt is much lower than with visible lasers and may be more beneficial for studies involving, for example, bioaerosols, where visible wavelengths may damage the specimens.

We make use of a $1064 \mathrm{~nm}$ fibre laser with a maximum power of $5 \mathrm{~W}$ (IPG Photonics). The power delivered to the trap is typically a few milliwatts, with power control by way of a half-wave plate and a polarising beam cube. We used a 100x oil immersion objective (Nikon CFI E Plan Achromat 100X oil, N.A. 1.25) to focus the beam into the sample chamber. This was identical to the objective used in our $532 \mathrm{~nm}$ work to allow a direct comparison. The aerosol droplets (for this and all the experiments detailed below) are created using an ultrasonic nebuliser (Omron U22(NE-U22-E)), which produces aerosols with a Mass Median Aerodynamic Diameter (MMAD) between approximately 3 and 5 microns. ${ }^{20}$ Custom glassware is attached to the nebuliser, allowing the droplets to be fed accurately into a sealed glass cube through an inlet hole, which acts as our trapping chamber. It is worth pointing out that the length and diameter of the glass piece used to couple the aerosols from the nebuliser into the trapping chamber plays a critical role in producing a flow of aerosols at the correct velocity and

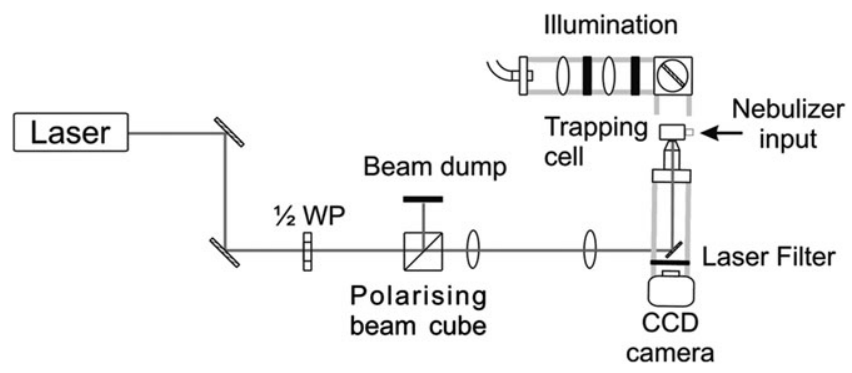

Fig. 1 Standard aerosol tweezers setup. 


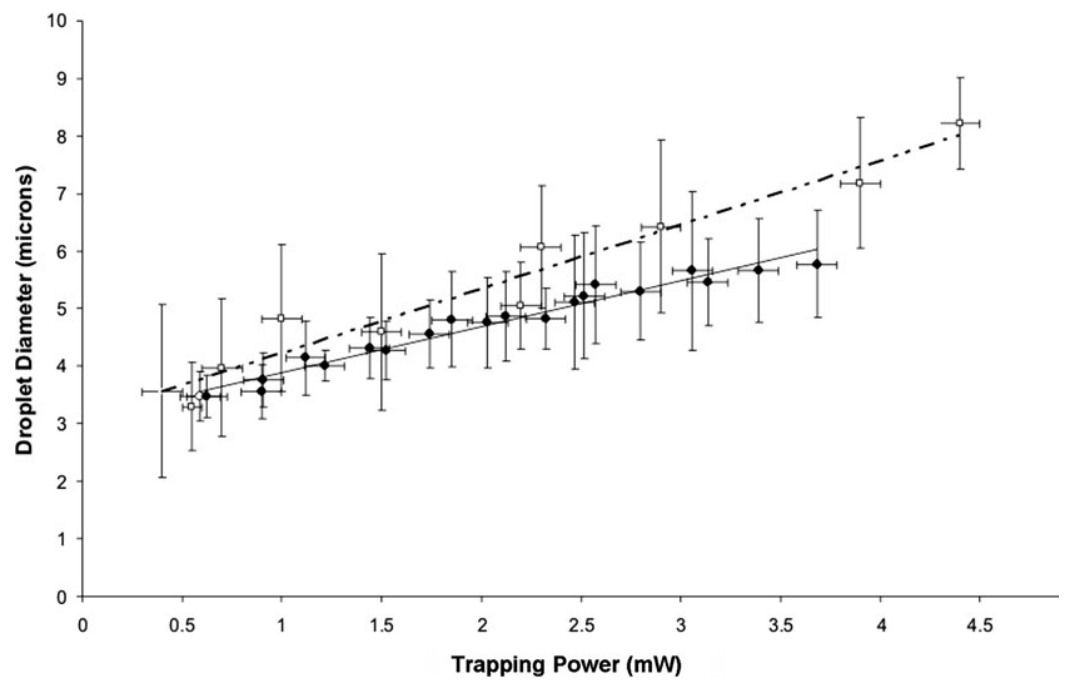

Fig. 2 Graph of droplet diameter against trap power for $2 \% \mathrm{w} / \mathrm{v}$ salt water solution at $532 \mathrm{~nm}$ (lower, solid trace) and $1064 \mathrm{~nm}$ wavelengths (upper, dashed trace). The points represent the mean diameter of droplets trapped over a range of experimental runs. The error bars give the standard error. The error bar in the $y$-direction gives an indication of the range of droplet sizes that can be trapped for a given power.

size into the chamber to allow trapping to occur. We have, however, not quantified this process and, while it is important, we can only state that for our nebuliser we use a glass tube of $5 \mathrm{~cm}$ in length with a $1.5 \mathrm{~cm}$ diameter, which starts to taper down to a diameter of $2 \mathrm{~mm}$ after $2.5 \mathrm{~cm}$. The droplets that we find in the trapping chamber that we typically trap tend to be in the size regime $2-15$ microns in diameter, the larger size being produced by coagulation. We measure the droplet diameter via calibrated video microscopy, which has shown to be a reliable method for estimating droplet size. ${ }^{12}$

We measured the power required to trap aerosols of a given size and also the $Q$ values. The $Q$ values are measured by trapping a droplet and then reducing the trapping power until it falls out. We can then use eqn (4) to find the $Q$ :

$$
Q=\frac{c}{n_{\mathrm{m}}} \frac{F}{P}
$$

Where $F$ is the force applied by the laser beam at power $P$. For a typical 6 micron diameter droplet, we find $Q \sim 0.25$, which is in keeping with our $532 \mathrm{~nm}$ work, but is lower than previously reported work. ${ }^{7}$ The smallest droplets of water that we have been able to trap are $\sim 3 \mu \mathrm{m}$ in diameter and need $\sim 0.5 \mathrm{~mW}$ minimum trapping power to be held. A full droplet size (water droplets with $2 \% \mathrm{w} / \mathrm{v}$ sodium chloride) versus trapping power plot is shown in Fig. 2, comparing $1064 \mathrm{~nm}$ and $532 \mathrm{~nm}$. We would expect slightly larger droplets to be trapped for a given power using the green light, but this is not observed, although within experimental error the graphs are very similar. The reason for this slight discrepancy is probably due to slight differences in the optics, trapping chambers and overall experimental setups.

Importantly, by controlling the sample relative humidity within the chamber we can observe stable long term trapping over periods of hours using infrared beams.

We anticipate that other liquids such as ethanol and dodecane should also be straightforward to trap using infrared light and this will be reported in a future publication. 


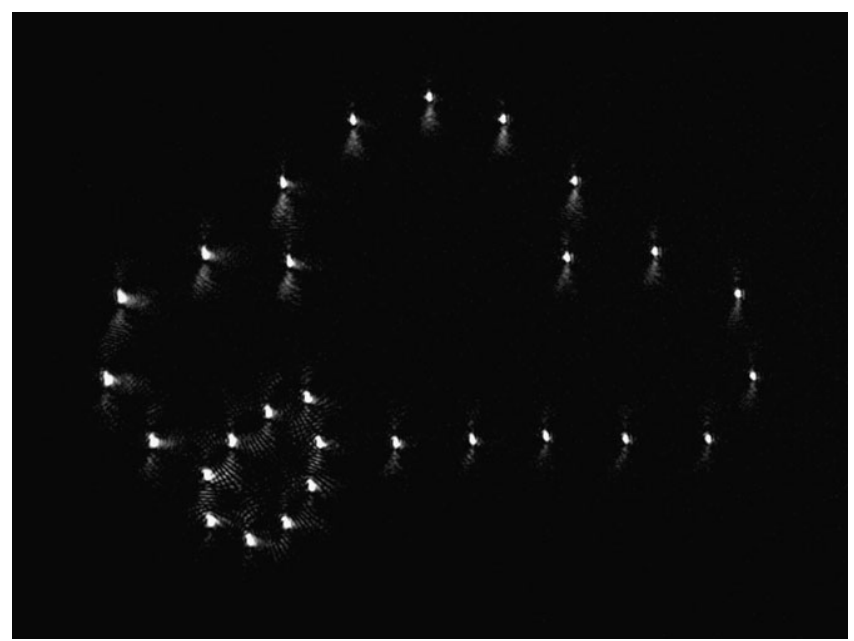

Fig. 3 Illustration of optical trap sites produced by a HOT. Note, the image has had some contrast enhancement to improve visibility.

\section{Holographic optical trapping}

A single beam optical tweezers system is a very powerful tool that can be used for many studies, but it is particularly useful in airborne studies to have the ability to carry out coagulation of droplets, and also to be able to carry out comparative studies on droplets simultaneously. There are many multibeam techniques that are able to trap and hold many particles simultaneously. The simplest is the dual beam optical tweezers, ${ }^{21}$ which was first used in the context of airborne particles by Hopkins et al. ${ }^{8}$ to fuse two water droplets. Other techniques include time-sharing traps using an Acousto-Optical Deflector (AOD) to rapidly move a beam between trapping sites. ${ }^{22}$ The beam must be moved rapidly enough to ensure that the droplets do not diffuse away or fall too far due to gravity in the time taken to scan the beam between them. ${ }^{12}$ Another approach, which we outline below, is to make use of Holographic Optical Tweezers (HOTs).

HOTs simply modify the phase of the initial laser that produces the trap and alter it into a desired intensity pattern. The devices used to modulate the phase are typically liquid crystal Spatial Light Modulators (SLMs). ${ }^{23-25}$ The patterns that can be generated can be as simple as two spots or be a complicated array as in Fig. 3. For most trapping work, these arrays of spots are sufficient, but continuous patterns can be generated, ${ }^{26}$ as can other beam types, such as Laguerre-Gaussian beams, ${ }^{27}$ which are discussed below. The patterns can be produced using various algorithms, ${ }^{28}$ which vary in their complexity depending on the amount of information that is known about the desired phase and whether spots or continuous patterns are required. Moreover, we can control optical aberrations of the traps by changing the applied holograms to correct for aberrations in the optical train, Fig. 4.

We recently demonstrated the first use of HOTs applied to aerosols, ${ }^{13}$ in which we showed that one could trap multiple aerosol water droplets simultaneously and then control their coagulation, illustrated in Fig. 5.

Our system makes use of a Holoeye LC-R 2500 SLM to modulate the phase of $532 \mathrm{~nm}$ continuous wave light from a Laser Quantum Finesse laser (maximum output $4 \mathrm{~W}$ ). We first rotate the laser's plane of polarisation to optimize the efficiency of the phase modulation, then, having expanded the beam using a telescope, the laser is incident on, and covers, the SLM. Two $4 \mathrm{f}$ imaging systems are placed directly after the SLM to reduce the beam size to slightly overfill the back aperture of the microscope objective (Nikon CFI E Plan Achromat 100X oil, N.A. 


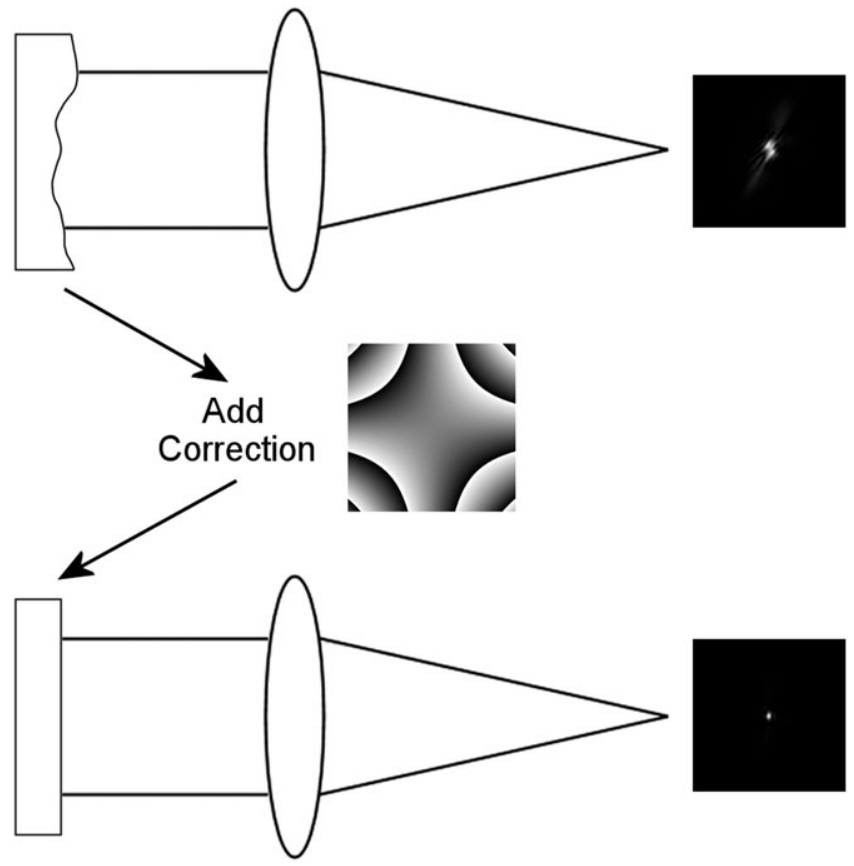

Fig. 4 Illustration of the way in which the SLM can be used to correct optical aberrations. The upper figure shows an uncorrected spot at the focal plane of the lens formed by an incoming aberrated waveform. The correcting hologram is shown in the central inset and the corrected spot in the bottom right.

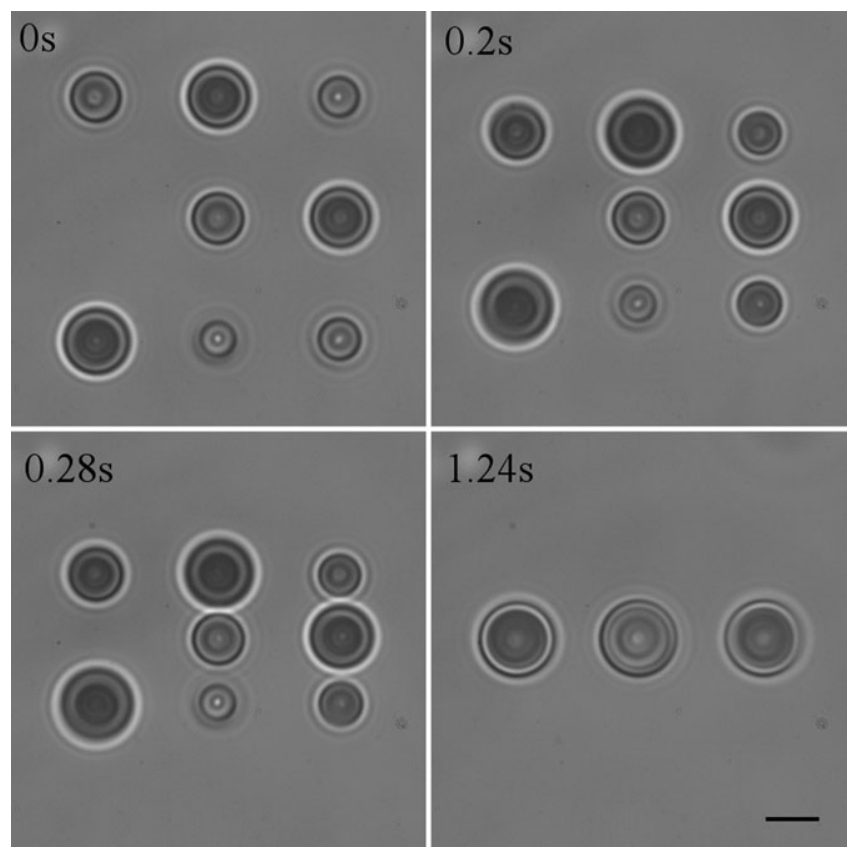

Fig. 5 Coagulation of water droplets using HOTs. The eight droplets trapped in the first image are coagulated into three droplets in around $1 \mathrm{~s}$. The scale bar is 5 microns. 


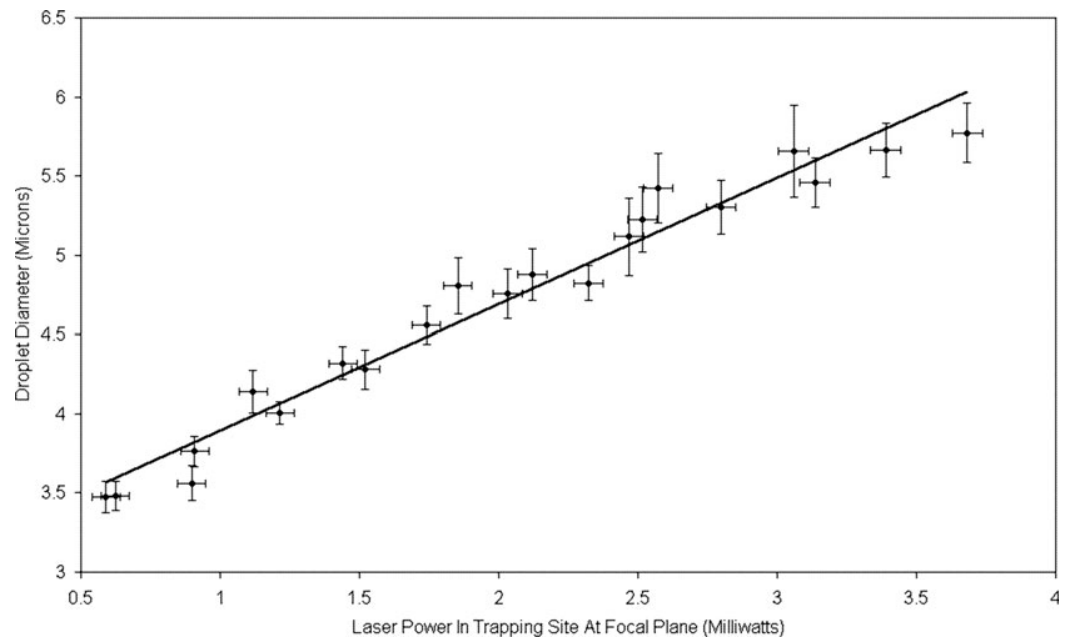

Fig. 6 Trapped droplet size versus power in a $532 \mathrm{~nm}$ optical tweezers. The points represent the mean diameter of droplets trapped over a range of experimental runs. The error bars give the standard error. The error bar in the $y$-direction gives an indication of the range of droplet sizes that can be trapped for a given power.

1.25). The 4f imaging systems also make the SLM and back aperture planes conjugate. Having focused the light into the trapping plane, the same objective is used for imaging. We generate our holograms making use of a custom LabVIEW program implementing an adaptive-additive algorithm that treats the SLM as a secondary microdisplay.

One of the key results of this work was a measurement of the trapping parameters of a single droplet held in an optical trap. The findings are illustrated in Fig. 6. The error bars in the $y$-direction are an indication of the range of sizes that can be trapped at a given power. The points on the graph are the mean droplet diameter and the error bars the standard error calculated over a number of experimental runs. The graphs indicate that at higher powers it is harder to trap smaller droplets. We do not have experimental evidence as to why this is but we believe it is due to the random trajectories that the droplets move in on their way to the trap. At higher powers the droplets feel a radiation pressure force far from the trap which makes it hard for them to get into the region where the gradient force dominates. Only droplets moving near the plane in which the focal region of the laser resides have a high probability of making it into the trapping region. As we increase the power, it becomes harder for the droplets to avoid the increasing radiation pressure zones around the trap focus. This effect is also evident from the graph in Fig. 2 above, showing the trapping ability of IR optical tweezers.

A second phenomenon that is observed is that if a droplet is trapped at a low power, then turning up the power leads to rapid particle oscillations and eventually the particle falls out of the trap. This is not behaviour normally associated with particles that are trapped in liquid. Why this should be the case is not yet fully clear, but is likely to be associated with a change in the dynamical regime in which it is trapped (i.e. a shift from overdamped to underdamped). ${ }^{17}$

The use of holograms can also be used to explore the transfer of angular momentum to airborne particles. To do so we make use of Laguerre-Gaussian beams, which carry Orbital Angular Momentum ${ }^{29}$ (OAM) due to the helical nature of their phase fronts. Such beams can be characterised by an azimuthal winding number $l$ and they carry an orbital angular momentum of $l$ h per photon, ${ }^{30}$ and this can be transferred to a particle trapped within the beam. We have shown rapid rotation rates are possible using $l=80$ beams in air, illustrated in Fig. 7 . 

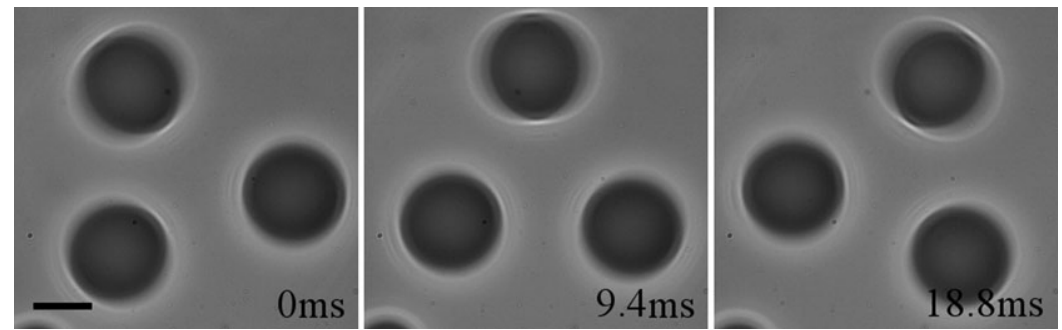

Fig. 7 Rotation of water aerosol using Laguerre-Gaussian beams. The three droplets are trapped in a single beam. The rotation is clearly seen as a function of time. Full rotation of complete circuits is possible but not shown. Scale bar is $5 \mu \mathrm{m}$.

\section{Dual beam fibre trapping of aerosols}

The idea of a dual beam trap goes back to the early days of optical trapping, ${ }^{15}$ where two focussed Gaussian beams were used to trap particles. This technique was developed by Constable et al. ${ }^{31}$ to make use of dual beams emerging from two aligned optical fibres. The trapping mechanism is due to radiation pressure and the advantage over conventional optical tweezers is that the beams are not delivered by high NA optics and can, if necessary, trap much larger particles than can be normally accessed by optical tweezers. Work by Kishan Dholakia's group in St. Andrews has shown optical manipulation of $0.1 \mathrm{~mm}$ diameter objects, for example. ${ }^{32}$

In the context of airborne trapping, we envisage the advantages of dual beam trapping to be the ability to trap a range of particle sizes not accessible to optical tweezers, increased probability of trap loading (i.e., better particle sampling capabilities) and also providing a more robust platform to trap particles in hostile environments (namely environments outside the safe, well controlled optical lab). This might allow us to build an engineered optical trapping and sampling device capable of being used in the field.

To this end, we have examined the possibility of trapping water aerosols using a dual beam fibre trap (which have far lower trapping power requirements than a recent dual beam trap making use of focussed laser beams ${ }^{33}$ ).

Coarsely, we can categorise optical fibres into two types, multimode and single mode. We envisage single mode fibre to be the better choice for this type of trap, but to simplify laser coupling, power requirements and cost, we restrict ourselves to multimode fibre here.

Our experimental setup consists of a $532 \mathrm{~nm}$ diode-pumped solid state laser (a $4 \mathrm{~W}$ maximum power Laser Quantum Finesse) which is coupled into two multimode optical fibres (0.22 NA multimode Vis-IR step index fibre) (item\#Afs50/125Y Thorlabs) with a 50 micron diameter core. We use a half-wave plate placed before a polarising beam cube to divide the power equally into the two arms of the system, where each beam is then coupled, via a fibre coupler, into a fibre. The half waveplate also allows us to control the relative power going into each arm of the device. A schematic of the experimental system is shown in Fig. 8.

The ends of the fibres are connectorised using ST connectors. We initially tried to make use of non-connectorised fibres but we found that they were very brittle, and even gentle air currents or knocks to the system could move the fibres out of alignment. Also, as we don't directly protect the ends of the fibres from the aerosols, it meant that we needed to repeatedly re-cleave the ends of the fibres after a given experimental run. Using the connectorised fibres makes them easier to clean, keeps them in more rigid alignment (by using standard optical mechanical mounts to hold them) and generally makes the system more robust.

The fibre ends are placed approximately 300 microns apart and are enclosed by a custom-made glass sheath that covers both the fibre ends and has inlet hole in the side to allow the aerosols to enter. A model of the trapping region is shown in Fig. 9. 


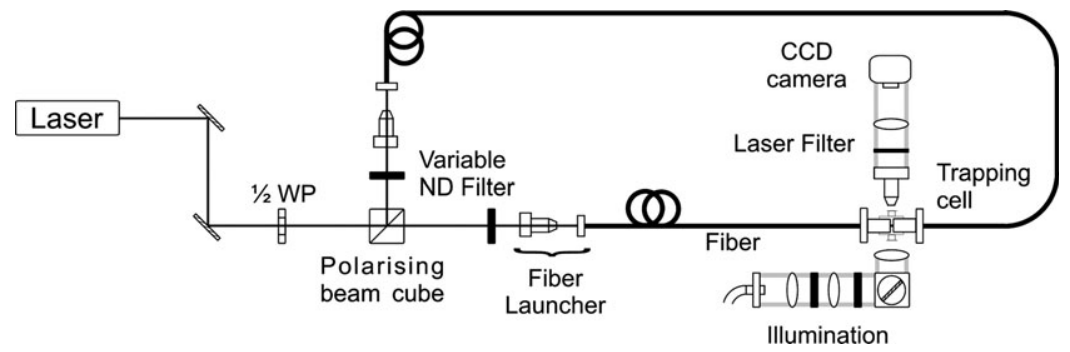

Fig. 8 Schematic of the dual beam fibre trap experimental setup.

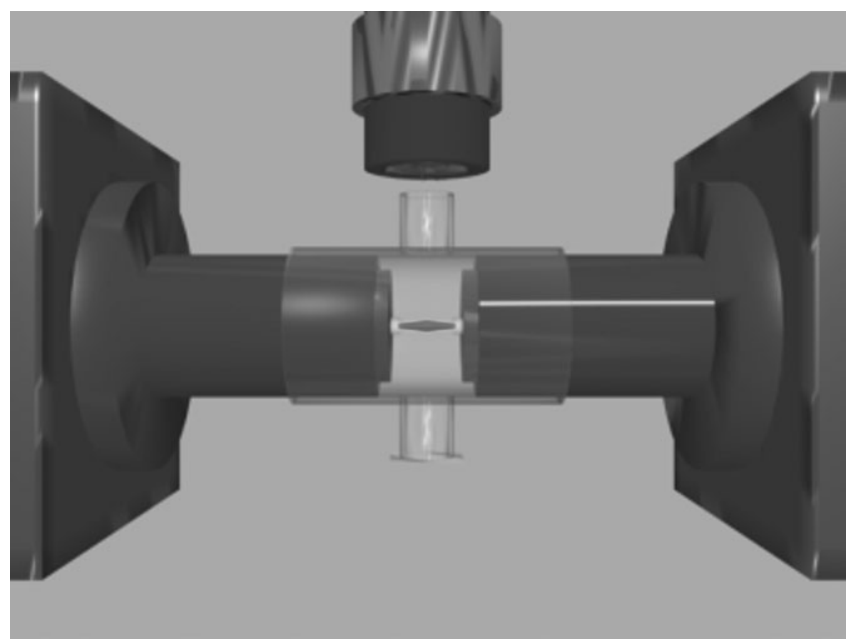

Fig. 9 Model of the dual beam fibre trap and the trapping region. The fibre ends (just visible as white insets into the transparent chamber) enter the transparent chamber, which acts to cut out air currents. The long working distance microscope objective is shown above the chamber. The chamber projections towards and directly opposite the objective are flattened windows to allow imaging into the chamber.

We find that the air currents are a significant factor in trying to trap the droplets. This is also true for the optically tweezed droplets, but here the geometry of the system coupled with the multimode nature of the light coming out of the fibre makes trapping slightly less robust. We hope improved engineering of the device will improve the stability - indeed, the work we have already done to make the device more rigid has improved our ability to trap significantly.

We make use of a long working distance microscope objective (Mitutoyo M Plan NIR 50x, NA 0.42) to observe the droplets in the trapping region due to the difficulty of using standard microscope objectives with short working distances to image beyond the ends of the connectorised fibres. An image of a trapped droplet is shown in Fig. 10.

We find that with using the multimode fibre, we are able to trap only a narrow range of droplet sizes as a function of power, in the range 3-7 microns, with an average size of approximately 5 microns. The gradient of the graph, Fig. 11, is low while the error bars are high, indicating that we do not gain much by increasing the power. To trap these droplets, we need a minimum of around $100 \mathrm{~mW}$ in each arm of the trap. We have observed trapping with $40 \mathrm{~mW}$ in each arm but this is less stable. While such powers are significantly higher than the optical tweezers discussed above, they are much lower than in a previously reported dual beam trap used to trap aerosols using focussed laser beams. ${ }^{33}$ While the amount of power does not seem to 

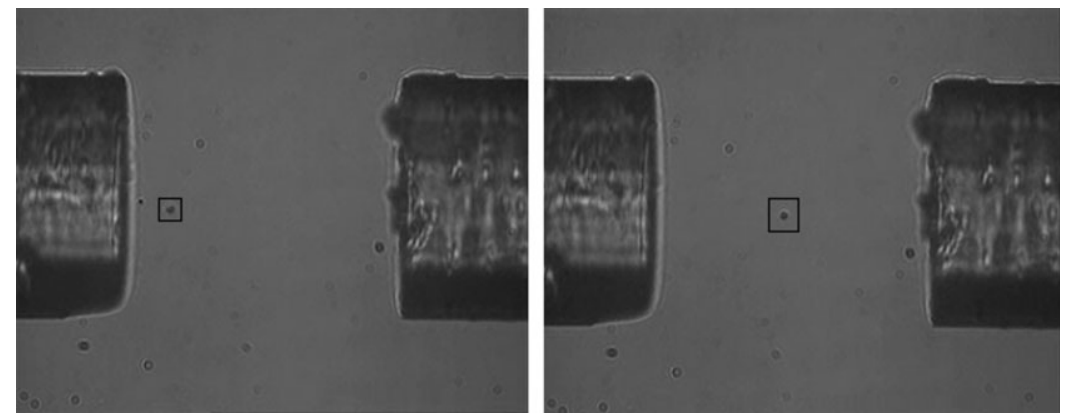

Fig. 10 Images of droplet trapped in fibre trap. The droplets in the trap are surrounded by a square box. The two images also show the droplet being guided with the initial trapping position on the left and then a new trapping position (right) about 50 microns from the initial position is reached by changing the power in one arm of the trap.

affect the droplet in any way and does not pose a constraint on the system, we anticipate that lower powers will be required to avoid heating and evaporation in certain circumstances. Lower trapping powers should be possible by altering the fibre separation and by using single mode fibres.

The use of multimode fibre makes a theoretical analysis of the system difficult due to the presence of numerous 'hotspots' in the intensity profile, resulting in the observation of ill-defined equilibrium positions in contrast to what one would expect in a single mode fibre trap. ${ }^{34}$ Thus, single droplets are found to be trapped at nearly any position between the fibre ends. We can, however, move the droplets between the fibres by changing the relative power provided to each fibre. For example, we can move the droplets up to 50 microns, as shown in Fig. 10.

We find that multiple droplets can be trapped within the trapping region due to the presence of multiple hot spots. This process is largely uncontrollable and does not constitute any form of optical binding ${ }^{35}$ which we expect to see when using single mode fibre. The use of hot spots does allow multiple droplets to be trapped in different horizontal planes, and it may be that in future work we can optimise this process to allow controllable trapping of multiple droplets, allowing parallel

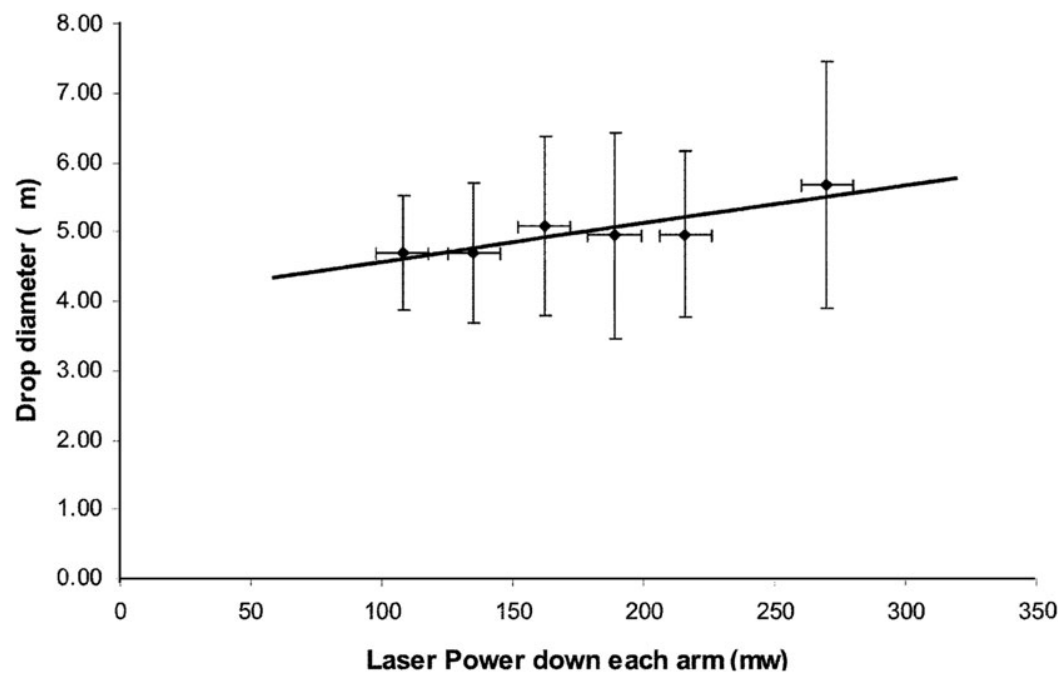

Fig. 11 Plot of trapped droplet size versus laser power in each arm for the fibre trap. The error bars indicate the standard error from the mean calculated over a number of experimental runs. 
processing of droplet composition, morphology and other chemical and thermodynamic processes.

\section{Optical guiding of aerosols}

We conclude our look at novel aerosol trapping techniques and technologies by considering a variant of the well established radiation pressure trapping technique, using a single beam to hold the particle against gravity. Here, we demonstrate that we can controllably guide a particle over $2.5 \mathrm{~mm}$ by making use of a novel beam known as a Bessel beam. ${ }^{36}$ A Bessel beam has the property that, when compared to a similar Gaussian beam, it does not diffract over an appreciable distance.

An ideal Bessel beam can be described by:

$$
E(r, f, z)=A_{0} \exp \left(i k_{z} z\right) J_{n}\left(k_{\mathrm{r}} r\right) \exp ( \pm i n f)
$$

where $J_{n}$ is a $n$ th-order Bessel function, $k_{z}$ and $k_{\mathrm{r}}$ are the longitudinal and radial wavevectors, with $k=\sqrt{k_{z}^{2}+k_{\mathrm{r}}^{2}}=2 p / l$ ( $\lambda$ being the wavelength of the electromagnetic radiation making up the Bessel beam) and $r, \phi$ and $z$ are the radial, azimuthal and longitudinal components, respectively. The intensity structure for a zeroth order Bessel beam is shown in Fig. 12.

For the Bessel beams considered here, $n=0$. Our Bessel beams are created using an axicon, ${ }^{37}$ or conical lens element. The opening angle of the axicon is given by:

$$
q=(n-1) g
$$

Where $n$ is the refractive index of the axicon material and $g$ is the opening angle of the axicon. As we cannot physically have a beam that is truly non-diffracting, the beam we generate can be considered quasi-non-diffracting over a given propagation distance. For the axicon-generated beam, we have a propagation distance given by:

$$
z_{\max }=\frac{k}{k_{\mathrm{r}}} w_{0} \gg \frac{w_{0}}{q} .
$$

In the following, we compare the guiding of water droplets using both a Bessel beam and a Gaussian beam. Our Gaussian beam has a beam diameter of $6 \mu \mathrm{m}$ and the Bessel beam has a central core diameter of $4 \mu \mathrm{m}$ and a propagation of $4 \mathrm{~mm}$. The core was found to expand to $25 \mu \mathrm{m}$ at the end of the $4 \mathrm{~mm}$ propagation distance, at which the 2nd ring became indistinct and the central spot became irregular.

A $2 \mathrm{~W} \mathrm{CW}$ ytterbium fibre laser at $1064 \mathrm{~nm}$ (IPG Photonics) was used to produce the Gaussian and Bessel beams. For the Gaussian beam experiment the beam is collimated with an appropriate beam waist so as to form the desired spot size after being focused with a final $f=25 \mathrm{~mm}$ lens. The Bessel beam is made using an axicon

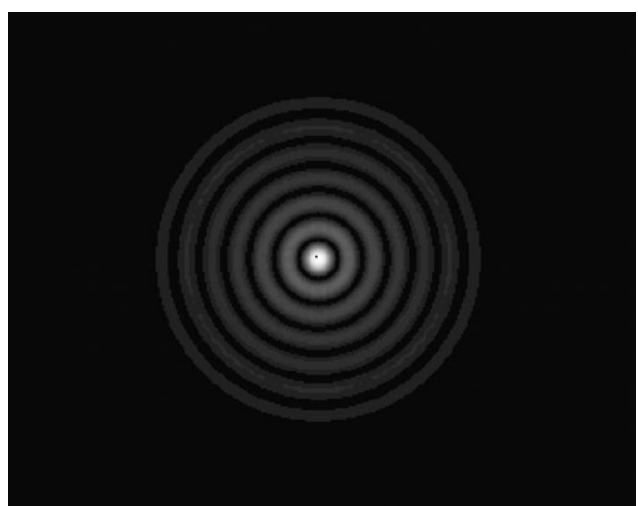

Fig. 12 Bessel beam intensity profile. 


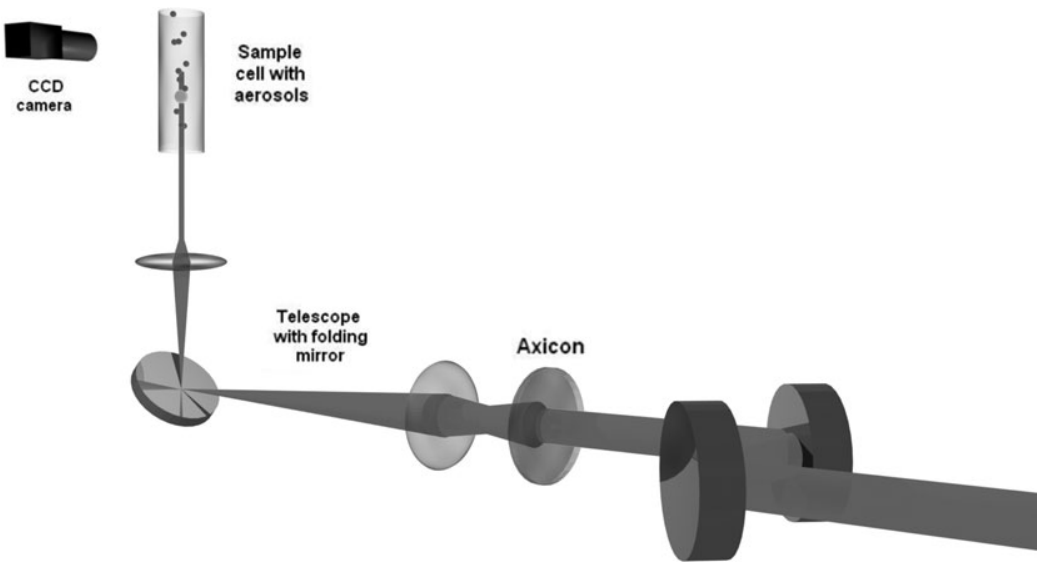

Fig. 13 Experimental setup for optical levitation using a Bessel beam.

in a double telescope arrangement. ${ }^{38}$ The first telescope expands the beam to allow adjustment of the propagation distance of the beam, while the second telescope is used to adjust the central core size of the beam (to match the spot size of the Gaussian beam). The aerosols are generated using a nebuliser attached to a nitrogen tank. The nitrogen flow into the nebuliser is controlled by a mass flow controller. The experimental arrangement is shown in Fig. 13 for the Bessel beam.

In order to trap the droplets we had to start with a high initial power $(\sim 1 \mathrm{~W}$ for the Gaussian beam, $200 \mathrm{~mW}$ in the Bessel beam core). Low powers (100 $\mathrm{mW}$ for the Gaussian, $>10 \mathrm{~mW}$ for the Bessel beam core) could be used to hold particles once they were captured, but were insufficient to trap the droplets moving under gravity and in the air currents in the sample chamber, which in this experimental arrangement were more of an issue than when using the optical tweezers to trap. Thus, we could trap a droplet with a high power and then subsequently guide it up and down by varying the power.

Using the Gaussian beam, water was stably trapped and could be made to move up and down with slow adjustment of the output power. The maximum guiding distances was found to be $250 \mu \mathrm{m}$ for water (Fig. 14).

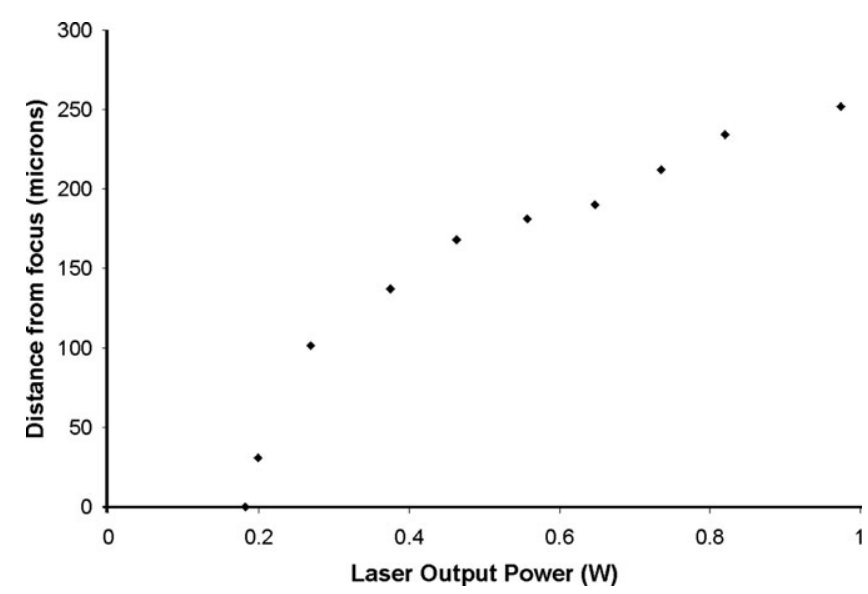

Fig. 14 Water droplet height against laser power (W) for water levitated in a Gaussian laser beam for a constant flow rate of nitrogen into the nebuliser of 0.58 litres $\mathrm{min}^{-1}$. The particle displacement is relative to the position for maximum power achieved at that flow rate. 


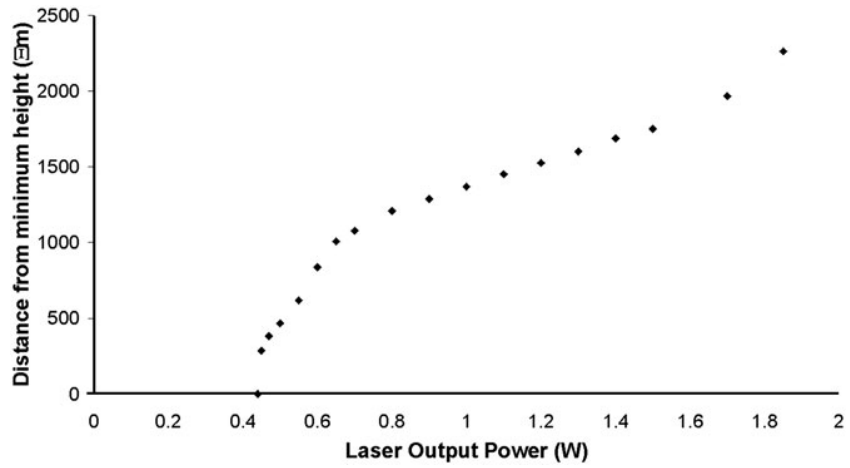

Fig. 15 Particle height against laser power (W) for water at a constant flow rate in a Bessel beam. The particle displacement is relative to the position for maximum power achieved at that flow rate.

We then performed similar experiments with the Bessel beam. The problem of trapping multiple droplets was a greater issue for the Bessel beam trap. Isolating single droplets was particularly difficult and the flow rate had to be carefully selected to avoid trapping multiple droplets. In some cases, droplets were trapped over a significant portion of the beam, forming extended arrays of particles. This was possible due to the relatively large vertical trapping region presented by the core over the large propagation distance. Another factor may have been the documented selfhealing effect of Bessel beams, which we have shown to form similar arrays in colloidal solutions. $^{39}$

For water droplets, the Bessel beam allowed guiding to occur over $2.5 \mathrm{~mm}$. This is approximately ten times the distance achieved in the Gaussian beam, illustrated in Fig. 15.

Further results for guiding ethanol and dodecane can be found in ref. 14

\section{Technologies and outlook}

Above, we have outlined a number of optical techniques that can be used to trap liquid aerosol droplets. Each of the techniques is relatively new and the full parameter space in which they operate has yet to be explored. However, it is clear that from a physics and technology development point of view that there are a number of interesting things to explore. Perhaps most importantly is the type of material that can be trapped optically, and the means to deliver that material into the trap. Most of the work discussed above made use of water droplets and the primary reason for this is that water is perhaps the most interesting liquid from an atmospheric point of view. However, we are largely limited to water and aqueous solutions due to our delivery method of an ultrasonic nebuliser, and organic materials, for example, damage the seals within our devices and cannot be regularly and controllably aerosolised. That is not to say that with alternative techniques these limitations cannot be overcome, and we are currently assessing alternative ultrasonic nebulisers that hold the liquids in a more compatible way.

Another question, and one motivation behind the fibre trap development, is whether we can trap non-liquid or non-transparent particles in such optical traps. This is of importance as many aerosols are not aqueous, black carbon being an important example. Clearly, we do not expect optical tweezers to be able to trap such particles but we are cautiously optimistic about the possibility of using a dual beam radiation pressure trap to trap objects that cannot be trapped in tweezers, either due to their optical properties or their size. One can, for example, trap much larger objects in a dual beam trap than in conventional fluid-based tweezers, as noted above. $^{32}$ 
To carry out more quantitative experiments on aerosols in optical traps we need to develop new, more controllable, ways to load the trap sites. Various options exist for loading techniques. We envisage a system design that makes use of techniques in atom optics where atoms are trapped in a 'dirty' chamber before being transferred through some mechanism to the 'science' chamber where the actual measurements are carried out. We believe that this can be achieved with aerosols either through sorting techniques, ${ }^{40}$ via guiding, as illustrated with the Bessel beams above, or via guiding through a physical object such as hollow core optical fibre. We are currently exploring these options to see if any will improve the general robustness of trapping or the specificity in what we can actually trap, i.e. improving the monodispersity of the aerosols in the science chamber.

More generally, optical manipulation techniques seem to offer a stable platform for the manipulation of small, micron-sized droplets, which are difficult to trap using other methods such as electrostatic (electrodynamic balances) or sonic traps. Damping would also seem to be better in optical traps, allowing the particles to be better localised. The optical techniques also lend themselves very well to integration with spectroscopic analyses, and with holographic techniques offer the ability to manipulate multiple particles simultaneously, which cannot be done in a controlled manner using other methods.

We conclude, then, with the optimistic comment that optical manipulation techniques can be put to good use in probing single and small numbers of aerosol droplets. There is, however, still much work to be done to understand the specific processes involved in trapping things in air using laser beams and in designing and engineering robust systems that can have a wide range of uses both in laboratory and field environments.

\section{Acknowledgements}

We would like to thank the Royal Society, EPSRC and NERC for funding the above work. DM is a Royal Society University Research Fellow, DRB and DR acknowledge EPSRC for supporting their PhDs, MDS is supported by an RSC/EPSRC Analytical Trust studentship. JB and ND thank the Nuffield Trust for support and SA was supported by a Royal Society of Edinburgh International Visitor award. We thank Jonathan Reid and Laura Mitchem for helpful discussions and advice.

\section{References}

1 A. Ashkin, J. M. Dziedzic, J. E. Bjorkholm and S. Chu, Opt. Lett., 1986, 11, 288.

2 K. Dholakia and P. Reece, Nano Today, 1, 18.

3 F. Ritort, J. Phys.: Condens. Matter, 2006, 18, R531.

4 D. G. Grier, Nature, 2003, 424, 810.

5 J. E. Molloy and M. J. Padgett, Contemp. Phys., 2002, 43, 241.

6 R. Omori, T. Kobayashi and A. Suzuki, Opt. Lett., 1997, 22, 816.

7 N. Magome, M. I. Kohira, E. Hayata, S. Mukai and K. Yoshikawa, J. Phys. Chem. B, 2003, 107, 3988.

8 R. J. Hopkins, L. Mitchem, A. D. Ward and J. P. Reid, Phys. Chem. Chem. Phys., 2004, 6, 4924.

9 M. D. King, K. C. Thompson and A. D. Ward, J. Am. Chem. Soc., 2004, 126, 16710.

10 L. Mitchem, J. Buajarern, R. J. Hopkins, A. D. Ward, R. J. J. Gilham, R. L. Johnston and J. P. Reid, J. Phys. Chem. A, 2006, 110, 8116.

11 L. Mitchem, J. Buajarern, A. D. Ward and J. P. Reid, J. Phys. Chem. B, 2006, 110, 13700.

12 J. Buajarern, L. Mitchem, A. D. Ward, N. H. Nahler, D. McGloin and J. P. Reid, J. Chem. Phys., 2006, 125, 114506.

13 D. R. Burnham and D. McGloin, Opt. Express, 2006, 14, 4175.

14 M. D. Summers, J. P. Reid and D. McGloin, Opt. Express, 2006, 14, 6373.

15 A. Ashkin, Proc. Natl. Acad. Sci. U. S. A., 1997, 94, 4853.

16 D. McGloin, Philos. Trans. R. Soc. London, Ser. A, 2006, 364, 3521.

17 R. Di Leonardo, G. Ruocco, J. Leach, M.J. Padgett, A. Wright, J. Girkin, D. Burnham and D. McGloin, submitted (cond-mat/0702557). 
18 J. P. Reid and L. Mitchem, Annu. Rev. Phys. Chem., 2006, 57, 245.

19 This was communicated in referee's comments to a grant proposal we submitted.

20 J. H. Dennis, C. A. Pieron and K. Asai, J. Aerosol Med., 2003, 16, 213.

21 E. Fallman and O. Axner, Appl. Opt., 1997, 36, 2107.

22 A. Resnick, Rev. Sci. Instrum., 2001, 72, 4059.

23 J. E. Curtis and D. G. Grier, Opt. Commun., 2002, 207, 169.

24 H. Melville, G. F. Milne, G. C. Spalding, W. Sibbett, K. Dholakia and D. McGloin, Opt. Express, 2003, 11, 3562.

25 G. Gibson, L. Barron, F. Beck, G. Whyte and M. Padgett, New J. Phys., 2007, 9, 1367.

26 D. McGloin, G. C. Spalding, H. Melville, W. Sibbett and K. Dholakia, Opt. Express, 2003, 11, 158.

27 R. M. Lorenz, J. S. Edgar, G. D. M. Jeffries, Y. Zhao, D. McGloin and D. T. Chiu, Anal. Chem., 2007, 79, 224.

28 G. Sinclair, P. Jordan, J. Courtial, J. Cooper and Z. J. Laczik, Opt. Express, 2004, 12, 6475 .

29 L. Allen, M. J. Padgett and M. Babiker, Prog. Opt., 1999, 31, 291.

30 L. Allen, M. W. Beijersbergen, R. J. C. Spreeuw and J. P. Woerdman, Phys. Rev. A, 1992, 45, 8185 .

31 A. Constable, J. Kim, J. Mervis, F. Zarinetchi and M. Prentiss, Opt. Lett., 1993, 18, 1867.

32 P.R.T. Jess, private communication.

33 K. Taji, M. Tachikawa and K. Nagashima, Appl. Phys. Lett., 2006, 88, 141111.

34 P. R. T. Jess, V. Garcés-Chávez, D. Smith, M. Mazilu, L. Paterson, A. Riches, C. S. Herrington, W. Sibbett and K. Dholakia, Opt. Express, 2006, 14, 5779.

35 N. K. Metzger, K. Dholakia and E. M. Wright, Phys. Rev. Lett., 2006, 96, 068102.

36 D. McGloin and K. Dholakia, Contemp. Phys., 2005, 46, 15.

37 J. H. MacLeod, J. Opt. Soc. Am., 1954, 44, 592.

38 J. Arlt, V. Garces-Chavez, W. Sibbett and K. Dholakia, Opt. Commun., 2001, 197, 239.

39 V. Garcés-Chávez, D. Roskey, M. D. Summers, H. Melville, D. McGloin, E. M. Wright and K. Dholakia, Appl. Phys. Lett., 2004, 85, 4001.

40 M. P. MacDonald, G. C. Splading and K. Dholakia, Nature, 2003, 426, 421. 